\title{
Single rare-earth ion spins in nanophotonic resonators
} Jonathan M. Kindem, Andrei Ruskuc, John G. Bartholomew, Jake Rochman, Yan Qi Huan, and Andrei Faraon

Kavli Nanoscience Institute and Thomas J. Watson, Sr., Laboratory of Applied Physics, Caltech, Pasadena, California 91125, USA Institute for Quantum Information and Matter, Caltech, Pasadena, California 91125, USA john.bartholomew@sydney.edu.au

\begin{abstract}
Rare-earth ions embedded in crystals are promising optically addressable spin qubits. We demonstrate this potential by measuring the optical and spin transition properties of single ${ }^{171} \mathrm{Yb}^{3+}$ ions coupled to nanophotonic resonators fabricated in $\mathrm{YVO}_{4}$. $\quad$ (C) $2020 \mathrm{The}$ Authors
\end{abstract}

\section{Introduction}

An ongoing challenge in quantum science and technology is to distribute entanglement by combining 'flying qubits', like photons, with 'stationary qubits' based on matter. The leading platforms to realise protocols for such quantum networks are atomic systems, either individually trapped or as ensembles [1], and solid-state optical centers [2]. The aim in all these systems is to realize stable optical transitions coupled to cavities for efficient interfacing with photons, and spin coherence lifetimes that are long enough to store quantum states during photon transit through the network.

Rare-earth ions in crystals are one class of solid-state emitters that have shown appeal for quantum networking applications. Previous experiments with rare-earth ion ensembles in crystals have demonstrated that they can possess narrow $(\sim 1 \mathrm{kHz})$, stable optical transitions and spin coherence times longer than the time it takes light to travel global distances $(\sim 100 \mathrm{~ms})$. However, repeating similar measurements with single ions is challenging because the long optical lifetimes $(100 \mu s \sim 10 \mathrm{~ms})$ of the relevant $4 \mathrm{f}$ energy levels result in low emission rates compared to other quantum emitters $[3,4]$.

In this work [5], we coupled the $984.5 \mathrm{~nm}$ transition of single ${ }^{171} \mathrm{Yb}^{3+}$-ions in a commercially available yttrium orthovanadate $\left(\mathrm{YVO}_{4}\right)$ crystal to a one-dimensional nanophotonic resonator (shown in Figure 1(b)). The resulting Purcell enhancement of the transition increased the photon emission rate by over two orders of magnitude (optical $\mathrm{T}_{1}$ in the cavity $=2.27 \mu \mathrm{s}$ ). We characterized the optical and spin transition properties of single ions at cryogenic temperatures between $40 \mathrm{mK}$ and $1.2 \mathrm{~K}$. In particular, we demonstrated optical coherence lifetimes (optical echo) $\mathrm{T}_{2}=1.6 \mathrm{~T}_{1}$, and spin coherence lifetimes up to $33 \mathrm{~ms}$.

a

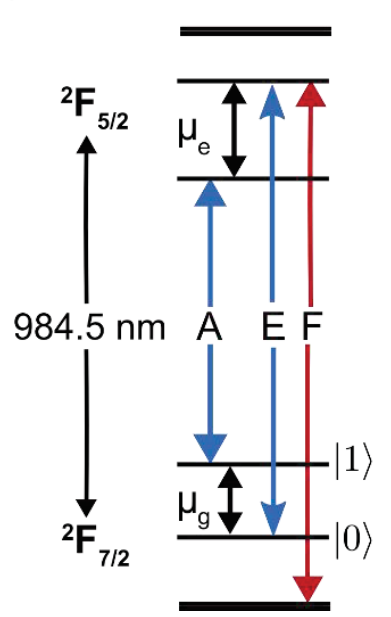

b

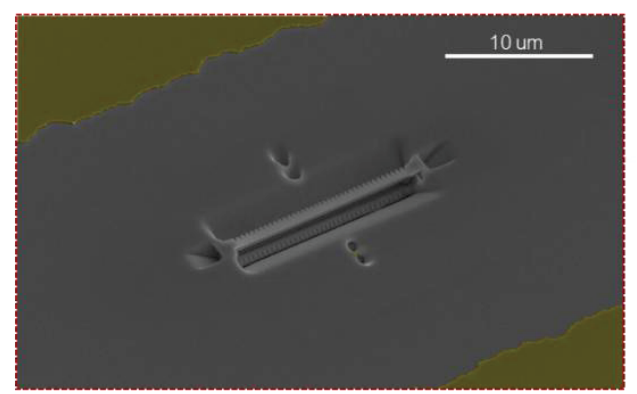

C

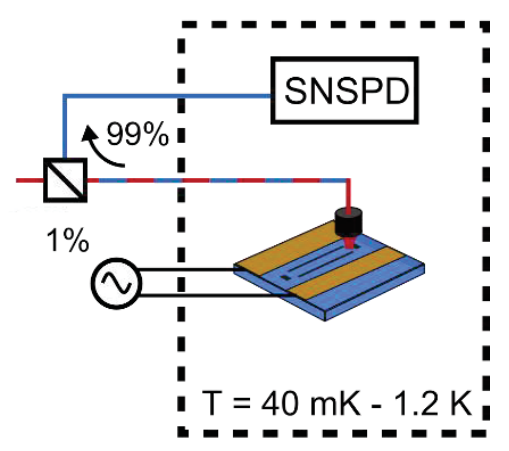

Fig. 1. (a) Zero-magnetic-field energy level structure for ${ }^{171} \mathrm{Yb}^{3+}$-ions in $\mathrm{YVO}_{4}$. Transitions A and E (blue) are coupled to the cavity mode, whereas $\mathrm{F}$ (red) is orthogonally polarized. We targeted the spin clocktransition between states $|0\rangle$ and $|1\rangle$ at a frequency $\mu_{g}=675 \mathrm{MHZ}$. (b) False-color scanning electron micrograph of the nanophotonic cavity located within a gold coplanar waveguide. (c) Schematic of the experiment. The device is maintained at a temperature below $1.2 \mathrm{~K}$ in a dilution refrigerator. We addressed the ion using optical excitation that is coupled to the device using a free space lens doublet, and microwave excitation that is coupled to a coplanar waveguide. Photon emission is collected back into a single mode fiber and detected by a superconducting nanowire single photon detector (SNSPD) in the same fridge. 


\section{Results and discussion}

a

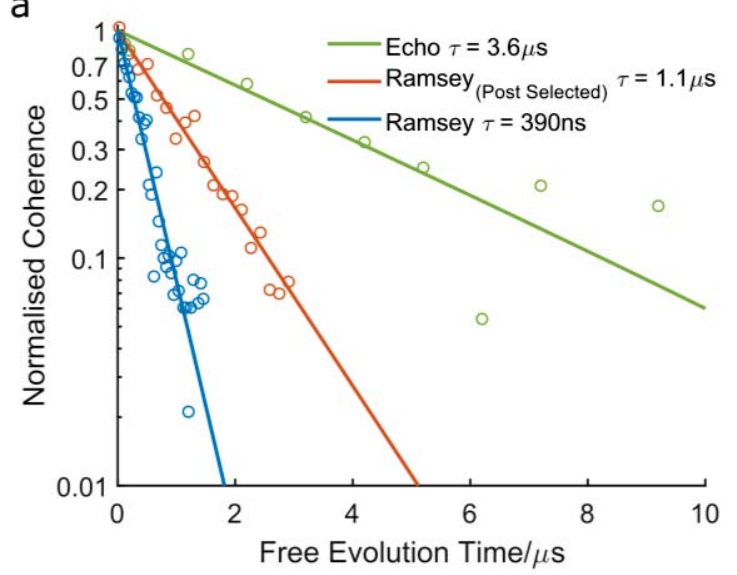

b

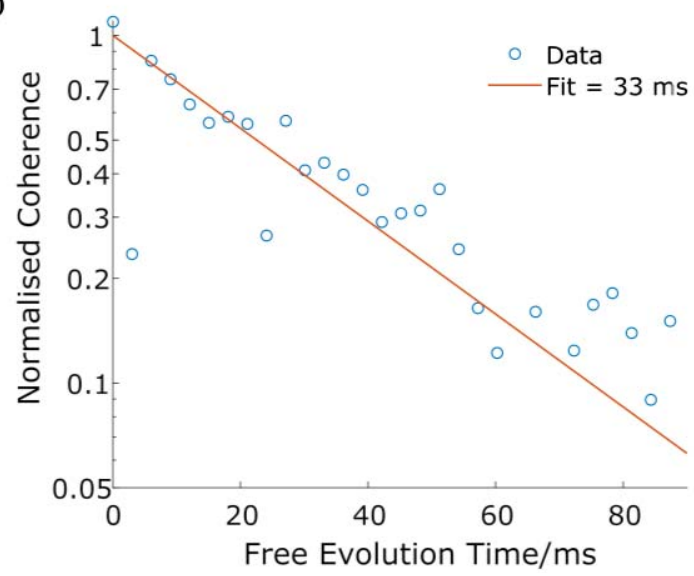

Fig. 2. (a) Single ${ }^{171} \mathrm{Yb}^{3+}$ ion optical coherence properties measured using Ramsey, post-selected Ramsey and echo pulse sequences. (b) Coherence lifetime measurements on the $\mu_{g}=675 \mathrm{MHz}$ spin transition using a Carr-Purcell-Meiboom-Gill pulse sequence.

Using pulsed excitation from a gated Ti:Sapph laser we isolated single ${ }^{171} \mathrm{Yb}^{3+}$-ions based on their frequencyresolvable optical transitions. Pulsed autocorrelation measurements yielded $g^{2}[0]<0.15$ confirming that we were addressing a single ion. Figure 2(a) shows the data from optical coherence measurements. The $\mathrm{T}_{2}{ }^{*}=390 \mathrm{~ns}$ measured by a Ramsey sequence was limited by quasi-static fluctuations impacting the optical transition frequency, which were mitigated by using post-selection. We also applied an optical $\pi$ pulse to rephase the quasi-static fluctuations and the resultant echo method yielded a significantly longer $\mathrm{T}_{2}$.

We also performed spin coherence lifetime measurements through pulsed microwave excitation at the ground-state spin transition frequency. We optically initialize the ion into state $|0\rangle$ or $|1\rangle$, apply microwave pulses at frequency $\mu_{g}$, and then read out the population of state $|1\rangle$ on the highly cyclic (>99\%) transition A (see Figure 1). With a single rephasing $\pi$ pulse we measure a $T_{2}>40 \mu$ s, which can be extended to greater than $30 \mathrm{~ms}$ through dynamic decoupling sequences as shown in Figure 2(b). This coherence lifetime remained constant at temperatures up to $1.2 \mathrm{~K}$. Factors limiting the coherence lifetime include contributions from the spin lifetime $\left(\mathrm{T}_{1}=50 \mathrm{~ms}\right)$ and residual magnetic dephasing arising from interactions with other spins in the host crystal.

In conclusion, we demonstrated single ${ }^{171} \mathrm{Yb}^{3+}$-ions in $\mathrm{YVO}_{4}$ nanophotonic cavities to be a promising optically addressable spin to realize light-matter interfaces for quantum networks. Future research will work to reduce spin impurities in the host crystal, and improve the cavity quality factor and light extraction efficiency to build toward multi-rare-earth ion networks.

\section{Acknowledgements}

This work was funded by a National Science Foundation (NSF) CAREER award (1454607), the AFOSR Quantum Transduction MURI (FA9550-15-1-002), NSF 1820790, and the IQIM, an NSF Physics Frontiers Center (PHY-1733907) with support from the Moore Foundation. The device nanofabrication was performed in the Kavli Nanoscience Institute at Caltech. The authors would like to thank Matthew Shaw, Sae Woo Nam, and Varun Verma for help with superconducting photon detectors, Alp Sipahigil for useful discussions, Keith Schwab for help with electronics, and Daniel Riedel for supporting measurements.

\section{References}

[1] Y. Yu et al., "Entanglement of two quantum memories via fibres over dozens of kilometres", Nature 578, 240 (2020).

[2] D. D. Awschalom, R. Hanson, J. Wrachtrup, and B. B. Zhou, "Quantum technologies with optically interfaced solid-state spins," Nat. Photon., 12, 516-527 (2018).

[3] T. Zhong et al., "Optically Addressing Single Rare-Earth Ions in a Nanophotonic Cavity”, Phys. Rev. Lett. 121, 183603 (2018).

[4] A. M. Dibos, M. Raha, C. M. Phenicie, and J. D. Thompson, "Atomic Source of Single Photons in the Telecom Band" Phys. Rev. Lett. $120,243601(2018)$ 
[5] J. M. Kindem et al., "Coherent control and single-shot readout of a rare-earth ion embedded in a nanophotonic cavity," https://arxiv.org/abs/1907.12161 (2019) 